IMECE2014-39612

\title{
SOUND TRANSMISSION LOSS THROUGH A CORRUGATED-CORE SANDWICH PANEL WITH INTEGRATED ACOUSTIC RESONATORS
}

\author{
Noah H. Schiller \\ NASA Langley Research Center \\ Hampton, VA, USA \\ Bart F. Zalewski \\ Zin Technologies, Inc. \\ Cleveland, $\mathrm{OH}, \mathrm{USA}$
}

\author{
Albert R. Allen \\ NASA Langley Research Center \\ Hampton, VA, USA \\ Benjamin S. Beck \\ National Institute of Aerospace \\ Hampton, VA, USA
}

\begin{abstract}
The goal of this study is to better understand the effect of structurally integrated resonators on the transmission loss of a sandwich panel. The sandwich panel has facesheets over a corrugated core, which creates long aligned chambers that run parallel to the facesheets. When ports are introduced through the facesheet, the long chambers within the core can be used as low-frequency acoustic resonators. By integrating the resonators within the structure they contribute to the static load bearing capability of the panel while also attenuating noise. An analytical model of a panel with embedded resonators is derived and compared with numerical simulations. Predictions show that acoustic resonators can significantly improve the transmission loss of the sandwich panel around the natural frequency of the resonators. In one configuration with $0.813 \mathrm{~m}$ long internal chambers, the diffuse field transmission loss is improved by more than $22 \mathrm{~dB}$ around $104 \mathrm{~Hz}$. The benefit is achieved with no added mass or volume relative to the baseline structure. The embedded resonators are effective because they radiate sound out-of-phase with the structure. This results in destructive interference, which leads to less transmitted sound power.
\end{abstract}

\section{INTRODUCTION}

This paper discusses an approach that could be used to improve the low-frequency transmission loss through a structure with no added mass. Low-frequency noise is notoriously difficult to attenuate without using bulky, heavy treatment. In aerospace vehicles, and even ground vehicles, there is a need for lightweight treatment options. Reducing interior noise is desirable for several reasons. In some vehicles, acoustic treatment is necessary to improve passenger and crew comfort. Other types of vehicles require acoustic treatment to attenuate high noise levels that would otherwise present a safety hazard to the crew or damage sensitive equipment.

Typically noise treatment either consists of porous absorbers or add-on acoustic resonators. Porous absorbers, such as fiberglass and foam, are usually light-weight materials that contain a network of interconnected cavities and channels. Porous absorbers work by converting the acoustic energy, in the form of pressure oscillations in the fluid, to heat. The dominant mechanism is typically viscous losses due to friction between the fluid and porous material. However, porous absorbers are not effective at low frequencies where the material thickness is much less than the acoustic wavelength [1]. As a result, this type of treatment is usually inadequate at frequencies below $300 \mathrm{~Hz}$ due to size and weight constraints. On the other hand, resonators can be effective at lower frequencies.

Resonators are dynamic devices that exhibit large oscillations at a particular frequency called the resonance frequency. Large pressure oscillations at the resonator inlet can have a significant impact on the acoustic environment. However the impact is limited to a relatively narrow frequency band around resonance. Despite this limitation, resonators are commonly used in many applications ranging from recording studios to jet engine nacelles [2]. Acoustic resonators have also been used in launch vehicle fairings to attenuate low frequency acoustic modes [3] and have been considered for use in propeller-driven aircraft to improve the transmission loss through the sidewall [4].

Most noise treatments, including acoustic resonators, take up space and add weight to the structure. Minimizing these two parameters is very important, particularly in aerospace applications. To address this issue for launch vehicle applications, Lane et al. [5, 6] proposed using a double-wall fairing with long rectangular chambers sandwiched between 1

This material is declared a work of the U.S. Government and is not subject to copyright protection in the United States. Approved for public release; distribution is unlimited. 
inner and outer facesheets. Ports were added to the inner facesheet to couple the chambers, or resonators, to the interior volume. A $1.51 \mathrm{~m}$ diameter cylindrical test article was built to evaluate the concept. The test article contained embedded resonators tuned to frequencies ranging from 60 to 2,000 $\mathrm{Hz}$. They found that the noise reduction, or difference between the average sound pressure outside and inside the cylinder, increased significantly when the resonators were active. $\mathrm{Li}$ and Vipperman [7] performed a thorough experimental characterization of a similar structure, and also showed promising results in terms of noise reduction.

This paper considers a similar structural concept, but focuses on transmission loss instead of noise reduction. The structure considered here is a sandwich panel with facesheets over a corrugated core, as shown in Figure 1. Corrugated-core structures such as this are currently used in automotive, aerospace, rail, shipbuilding, and building construction applications because they can have a very high stiffness-toweight ratio [8]. This paper considers the implications of using the long, hollow chambers within the core as integrated acoustic resonators. This can be achieved by adding circular ports to the bottom side of the panel which act as inlets to the resonators. The integrated resonators could be used to reduce interior noise without increasing the weight or size of the structure. Therefore the concept is applicable to a wide-range of light-weight structures that must meet interior noise requirements, including launch vehicle fairings and aircraft sidewalls.

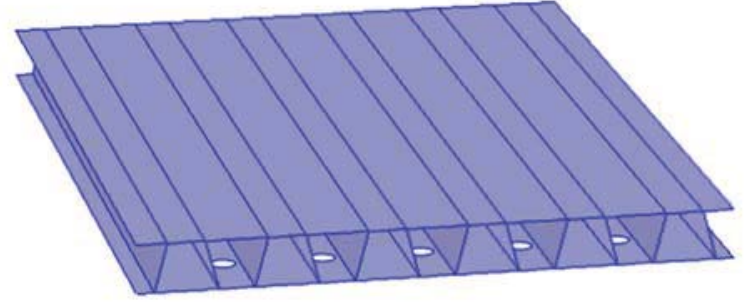

Figure 1: Corrugated-core panel with circular inlets.

The paper begins with the derivation of an analytical model for the transmission loss through a panel with embedded resonators. A numerical model of the corrugated-core panel is then described, followed by a comparison of the analytical and numerical predictions. The effect of embedded resonators on transmission loss is then demonstrated, along with a physical explanation of how they work. The following section describes the impact of several parameter variations, including chamber length, core geometry, and port size. Finally, some concluding remarks are provided.

\section{ANALYTICAL MODEL}

This section begins with an impedance model for lowfrequency acoustic resonators. A general transmission loss model for panels with embedded resonators is then presented. Finally, diffuse field transmission loss is discussed.

\section{Acoustic resonators}

The acoustic resonators embedded within the corrugatedcore panel consist of long trapezoidal cavities, as depicted in Figure 1, with an inlet perpendicular to the cavity on the lower facesheet. Since it has been shown that the effect of a $90^{\circ}$ turn is negligible at low frequencies [9], the resonators can simply be approximated as a long cavity with an inlet in one end. Based on a one-dimensional plane-wave analysis, the acoustic impedance of the cavity can be written as [10]:

$$
Z_{c}=-j \frac{\rho c}{A_{c}} \cot \left(k_{c} L\right)
$$

where $\rho$ is the density of the fluid, $c$ is the speed of sound, $A_{c}$ is the cross sectional area of the cavity, $L$ is the length of the cavity, and $k_{c}=\frac{\omega}{c}+(1+j) \alpha_{c}$ is the complex wavenumber. A wide-duct boundary layer absorption model is used to define the attenuation coefficient, $\alpha_{c}=\frac{1}{r_{c}} \sqrt{\frac{\omega \mu}{2 \rho c^{2}}}\left(1+\frac{\gamma-1}{\sqrt{P r}}\right)$, which accounts for the thermal and viscous losses in the cavity $[11,12]$. The remaining terms are defined as: $\omega$ is angular frequency, $r_{c}=\sqrt{A_{o} / \pi}$ is the effective radius of the cavity, $\mu$ is the dynamic viscosity of the fluid, $\gamma$ is the ratio of heat capacities, and $\operatorname{Pr}$ is the Prandtl number. For convenience, the relevant properties of air at $20^{\circ} \mathrm{C}$ and $1 \mathrm{~atm}$ are defined in Table 1.

Table 1: Properties of air at $20^{\circ} \mathrm{C}$ and $1 \mathrm{~atm}$.

\begin{tabular}{|c|c|}
\hline$\rho$ & $1.2 \mathrm{~kg} / \mathrm{m}^{3}$ \\
\hline$c$ & $343 \mathrm{~m} / \mathrm{s}$ \\
\hline$\gamma$ & 1.4 \\
\hline$\mu$ & $18.1 \times 10^{-6} \mathrm{~Pa} \mathrm{~s}$ \\
\hline$P r$ & 0.71 \\
\hline
\end{tabular}

The acoustic impedance of the inlet needs to be considered as well as the impedance of the cavity. If losses are neglected, the acoustic impedance of the inlet can be represented as $[10,12]$

$$
Z_{o}=j \omega M_{A}
$$

where $M_{A}=\rho L_{e f f} / A_{o}$ is the acoustic inertance of the fluid in the neck of the inlet, $A_{o}$ is the cross-sectional area of the inlet, and $L_{e f f}$ is the effective length of the neck. If the inlet is set in a thin wall, then $L_{\text {eff }}$ can be approximated as $\frac{\pi}{2} r_{o}[13,12]$, where $r_{o}$ is the radius of the circular inlet. The thermal and viscous losses in the inlet can be included by rewriting Eq. (2) using the complex wavenumber $k_{o}=\frac{\omega}{c}+(1+j) \alpha_{o}$, such that

$$
Z_{o}=j\left(k_{o} c\right) M_{A}+\theta_{r}
$$

where $\alpha_{o}=\frac{1}{r_{o}} \sqrt{\frac{\omega \mu}{2 \rho c^{2}}}\left(1+\frac{\gamma-1}{\sqrt{P r}}\right)$ is the attenuation coefficient based on the wide-duct boundary layer absorption model $[11,12]$. The second term in Eq. (3) accounts for the radiation losses at the inlet and is defined as $\theta_{r}=\rho c k^{2} / 2 \pi$ [13]. The combined acoustic impedance of the resonator can then be expressed as

2

This material is declared a work of the U.S. Government and is not subject to copyright protection in the United States. Approved for public release; distribution is unlimited. 


$$
Z_{r}=Z_{o}+Z_{c}=j k_{o} c \frac{\rho L_{\text {eff }}}{A_{o}}-j \frac{\rho c}{A_{c}} \cot \left(k_{c} L\right)+\theta_{r}
$$

It is useful to consider two special cases. First, consider the case where the inlet and cavity areas are equal. In this case, there is no inlet restriction and therefore $L_{e f f}=0$. This corresponds to a conventional quarter-wave resonator. If the effect of the thermal and viscous losses on the reactance of the cavity is neglected, the acoustic impedance of the quarter-wave resonator can be written as

$$
Z_{\lambda / 4}=-j \frac{\rho c}{A_{c}} \cot (k L)+\theta_{c}
$$

where $k=\omega / c$, and $\theta_{c}$ is a resistive term accounting for losses in the chamber and acoustic radiation. Resonance occurs when the reactance (i.e. imaginary part of the impedance) equals zero. Therefore the first resonance, or natural frequency, of the cavity can be written as:

$$
f_{\lambda / 4}=\frac{c}{4 L}
$$

For the second special case, it is assumed that the acoustic wavelength $\lambda$ in the fluid is much longer than the dimensions of the resonator. In this case a lumped parameter model is appropriate. To derive the lumped parameter model, notice that the term $\cot (k L)$ can be expanded as

$$
\cot (k L)=\frac{1}{k L}-\frac{k L}{3}-\frac{(k L)^{3}}{45}-\frac{2(k L)^{5}}{945}+\cdots
$$

If $k L$ is sufficiently small such that $L<\lambda / 16$, then $\cot (k L) \approx$ $\frac{1}{k L}[10]$. If the effects of the thermal and viscous losses on the reactance are again neglected, the acoustic impedance of the resonator given in Eq. (4) simplifies to the classic Helmholtz resonator equation [13]. Specifically, the impedance simplifies to:

$$
Z_{h}=j k c \frac{\rho L_{e f f}}{A_{o}}-j \frac{\rho c}{A_{c}} \frac{1}{k L}+\theta_{h}=j \omega M_{A}-j S / \omega+\theta_{h}
$$

where $M_{A}$ is the acoustic inertance, $S=\frac{\rho c^{2}}{V}$ is the acoustic stiffness, $V=A_{c} L$ is the volume of the cavity, and $\theta_{h}$ accounts for the resistive losses in the cavity and inlet. Helmholtz resonators are relatively easy to understand because of the direct mechanical analogy with a mass-spring system. In a conventional Helmholtz resonator, the slug of fluid in the neck acts as the mass, while the stiffness is related to the volume of fluid in the cavity. The natural frequency of the Helmholtz resonator can be calculated as:

$$
f_{h}=\frac{1}{2 \pi} \sqrt{\frac{S}{M_{A}}}=\frac{c}{2 \pi} \sqrt{\frac{A_{o}}{V L_{e f f}}}
$$

\section{Plane wave transmission loss through a panel with embedded resonators}

Next consider the oblique sound transmission through a non-porous, infinite, uniform panel with embedded resonators, as depicted in Figure 2. When an acoustic plane wave is incident on the panel, the transverse velocity of the panel can be written as [12]

$$
v=\frac{2 \widehat{p}_{l}}{2 z_{i}+z_{p}}
$$

where $\widehat{p}_{\imath}$ is the complex amplitude of the incident pressure wave, $z_{i}=z_{f}=\rho c /(\cos (\theta))$ is the local specific impedance of the fluid at the surface of the panel, $\theta$ is the propagation angle of the incident pressure wave as depicted in Figure 2, and $z_{p}$ is the specific impedance of the panel. On the opposite side of the panel,

$$
\widehat{p_{t}}=z_{t} v
$$

where the local specific impedance on the transmitted side, $z_{t}$, is a combination of the specific acoustic impedance of the fluid and the resonators. The impedances of the fluid and resonators are combined in parallel while taking into account the surface area of each as,

$$
z_{t}=\frac{1}{\frac{1-\sigma}{z_{f}}+\frac{\sigma}{z_{r}}}
$$

where $\sigma=N_{r} A_{o} / S$ is the ratio of the total resonator inlet areas divided by the total panel surface area, $N_{r}$ is the number of resonators in the panel, and $S$ is the surface area of the radiating side of the panel. Note that $z_{r}$ is the specific acoustic impedance of the resonator, not the full acoustic impedance $Z_{r}$ as defined in Eq. (4). The specific acoustic impedance can be found by multiplying acoustic impedance by the surface area of the inlet: $z_{r}=Z_{r} A_{o}$. The plane wave sound-power transmission coefficient can then be defined as

$$
\tau(\theta)=\left|\frac{\widehat{p_{t}}}{\widehat{p}_{l}}\right|^{2}=\left|\frac{2 z_{t}}{2 z_{i}+z_{p}}\right|^{2}
$$

and therefore the plane wave transmission loss is

$$
T L(\theta)=10 \log _{10}(1 / \tau(\theta))=10 \log _{10}\left(\left|\frac{2 z_{i}+z_{p}}{2 z_{t}}\right|^{2}\right)
$$

For the purposes of this investigation, the specific impedance of the panel is taken as $z_{p}=-j \omega m_{s}$, where $m_{s}$ is the mass per unit area of the panel. This is a good approximation for the specific impedance of an infinite panel at low frequencies, well below the critical frequency where the acoustic wavelength in air matches the bending wavelength in the structure. The nominal panel properties and resonator dimensions used in the analytical model are defined in Table 2.

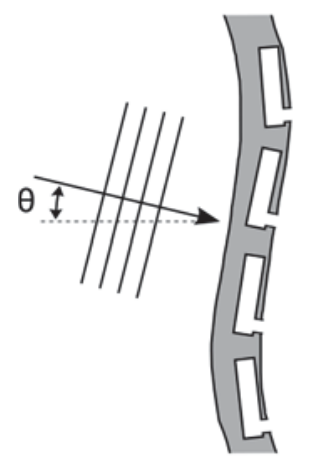

Figure 2: Panel with embedded resonators.

This material is declared a work of the U.S. Government and is not subject to copyright protection in the United States. Approved for public release; distribution is unlimited. 
Table 2: Panel properties and nominal resonator dimensions.

\begin{tabular}{|c|c|}
\hline Mass per unit area of panel, $m_{S}$ & $8.12 \mathrm{~kg} / \mathrm{m}^{2}$ \\
\hline Surface area of the panel, $S$ & $0.495 \mathrm{~m}^{2}$ \\
\hline Number of embedded resonators, $N_{r}$ & 11 \\
\hline Cavity length, $L$ & $0.718 \mathrm{~m}$ \\
\hline Cavity cross-sectional area, $A_{c}$ & $2.58 \times 10^{-3} \mathrm{~m}^{2}$ \\
\hline Inlet area, $A_{o}$ & $5.07 \times 10^{-4} \mathrm{~m}^{2}$ \\
\hline
\end{tabular}

\section{Diffuse field transmission loss}

A diffuse acoustic field implies that plane waves are incident from all angles with equal probability and random phase. The diffuse field transmission coefficient can be calculated from the plane wave equations given in the previous section as [14]:

$$
\tau_{d}=\int_{0}^{\pi / 2} \tau(\theta) \sin (2 \theta) d \theta
$$

and the diffuse field transmission loss can be calculated as

$$
T L_{d}=10 \log _{10}\left(1 / \tau_{d}\right)
$$

For this study the diffuse field transmission loss is estimated using 900 different angles of incidence equally distributed between 0 and 89.9 degrees.

\section{NUMERICAL MODEL}

A 3D finite element model was used to verify the accuracy of the analytical model. The modeled system is shown in Figure 3. The model includes two perfectly matched layer (PML) subdomains approximating infinite air domains. Periodic Floquet boundary conditions [15] are used on the lateral edges to model infinite periodic fluid and structural domains. A background pressure field is defined in the air domain above the panel. The pressure field approximates an incident plane wave propagating at an angle $\theta$ with respect to the plate normal. The corrugated-core sandwich panel is shown in blue. The overall size of the panel is $0.813 \mathrm{~m}$ by $0.610 \mathrm{~m}$. The panel contains 11 chambers, which are each $0.813 \mathrm{~m}$ long. The inlets to the acoustic resonators are modeled as circular ports with a radius of $0.0127 \mathrm{~m}$. The ports are centered 0.0826 $\mathrm{m}$ from the edge of the panel. Therefore the effective length of the resonators is $0.718 \mathrm{~m}$, which corresponds to the distance from the edge of the inlet to the far wall. The inlet locations are staggered on either side of the panel to reduce the interaction between neighboring inlets. Viscous losses in the core and inlets are captured using the wide-duct boundary-layer absorption model [11, 12].

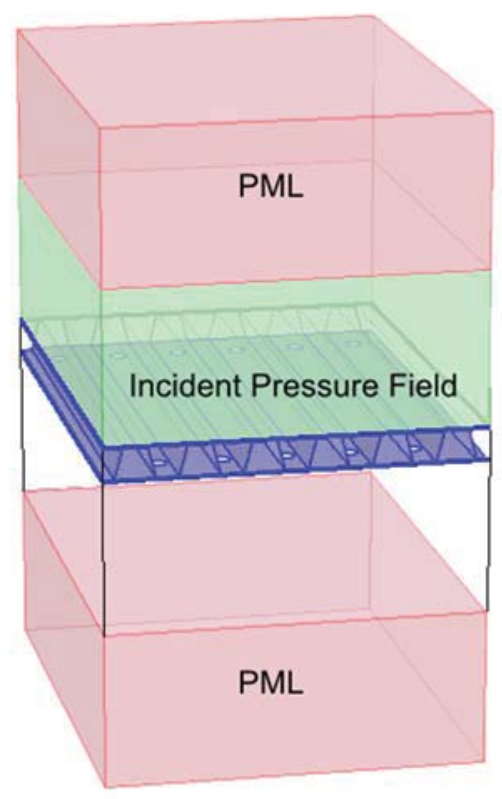

Figure 3: Acoustic and structural domains included in the numerical model.

An enlarged view of the panel cross-section is shown in Figure 4. The modeled panel has $1 \mathrm{~mm}$ thick facesheets over a corrugated core. The corrugated material is assumed to $0.5 \mathrm{~mm}$ thick. Therefore the thickness of the flanges, or regions where the core and facesheets overlap, is $1.5 \mathrm{~mm}$. The core is symmetric, such that the top and bottom flange widths are both $0.0381 \mathrm{~m}$. Because of the core symmetry, the volumes of all the resonators are identical. The overall panel thickness is $0.0508 \mathrm{~m}$ and the spacing is $0.102 \mathrm{~m}$. Both the facesheets and core are assumed to be made of aluminum and were modeled using shell elements. The material is assumed to have an elastic modulus of $70 \times 10^{9} \mathrm{~Pa}$, a density of $2700 \mathrm{~kg} / \mathrm{m}^{3}$, Poisson's ratio of 0.33 , and a structural loss factor of 0.01 .

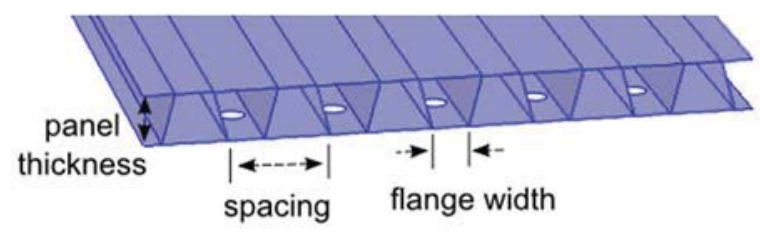

Figure 4: Panel cross section.

The transmission loss through the structure is calculated as:

$$
T L(\theta)=10 \log _{10}(1 / \tau(\theta))
$$

where the angle-dependent transmission coefficient is defined as $\tau(\theta)=\Pi_{t}(\theta) / \Pi_{i}(\theta)$. The incident power is calculated as $\Pi_{i}(\theta)=P_{i}^{2} \cos (\theta) /(2 \rho c)$, while the transmitted power, $\Pi_{t}(\theta)$, is found by integrating the time-averaged acoustic intensity over a plane below the panel.

Tetrahedral elements are used to mesh the acoustic domain. The maximum element size in the fluid is $84 \mathrm{~mm}$, however most elements are much smaller, particularly near the panel.

4

This material is declared a work of the U.S. Government and is not subject to copyright protection in the United States. Approved for public release; distribution is unlimited. 
The mesh is more than sufficient to resolve the shortest wavelengths in the elastic and acoustic domains through $140 \mathrm{~Hz}$, which is the upper frequency limit on the analysis. The model contains approximately 240,000 degrees of freedom, and it takes just over 30 minutes to run a direct frequency solve at 25 frequencies on a $2.20 \mathrm{GHz}$ quad-core processor.

\section{RESULTS AND DISCUSSION}

This section presents the results of the study. The section starts with a comparison of the analytical and numerical predictions. The sound transmission loss through a baseline corrugated-core panel (with no inlets) is then compared to the transmission loss through an identical panel with inlets. A physical explanation of the results is then provided. Finally, a parameter study is described, which summarizes the effect of parameter variations on the transmission loss of the system.

\section{Comparison between analytical and numerical models}

Figure 5 compares analytical and numerical predictions of the plane wave transmission loss through the corrugated-core panel with embedded resonators. While curves are only shown for two different angles of incidence, the comparison is similar at other angles as well. The solid lines correspond to the analytical predictions defined in Eq. (14) with the resonator impedance defined in Eq. (4). The dashed lines were generated using the detailed finite element model. Notice that the solid and dashed lines are nearly identical for both angles of incidence. This suggests that the assumptions underlying the analytical model are good. Specifically, it suggests that the chamber shape, elastic compliance of the facesheet and core, $90^{\circ}$ bend through the inlet, and interaction between resonators, are not important for this particular configuration. It also suggests that the panel can be accurately modeled as a limp mass at these frequencies.

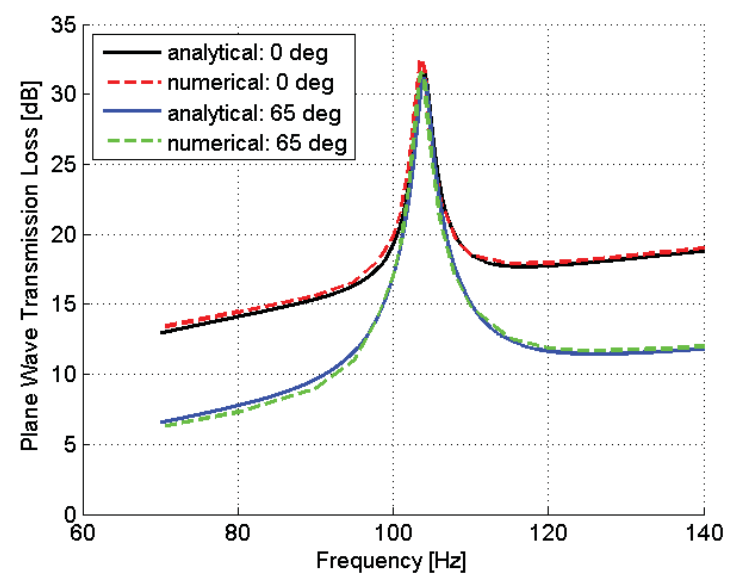

Figure 5: Plane wave transmission loss of the panel with embedded resonators at two different angles of incidence.

The excellent agreement between the two models suggests that similar configurations can be evaluated using the analytical instead of the numerical model with very little loss of accuracy. This is beneficial since analytical predictions are 3.5 orders of magnitude faster than numerical predictions. Therefore, unless otherwise stated, subsequent predictions presented in this paper are generated using the analytical model.

\section{Sound transmission loss with and without embedded resonators}

Figure 6 shows the plane wave transmission loss at three angles of incidence. The solid lines correspond to the baseline corrugated-core sandwich panel without inlets. In this case the acoustic cavities in the core do not behave like acoustic resonators and therefore the response is controlled primarily by the mass of the structure. Notice that the curves increase smoothly with frequency as expected for a panel in the masscontrolled region. Also notice that the transmission loss is angle dependent. Specifically, transmission loss is largest when the acoustic waves are normal to the panel's surface and decreases as the angle between the surface normal and wave propagation direction approaches 90 degrees.

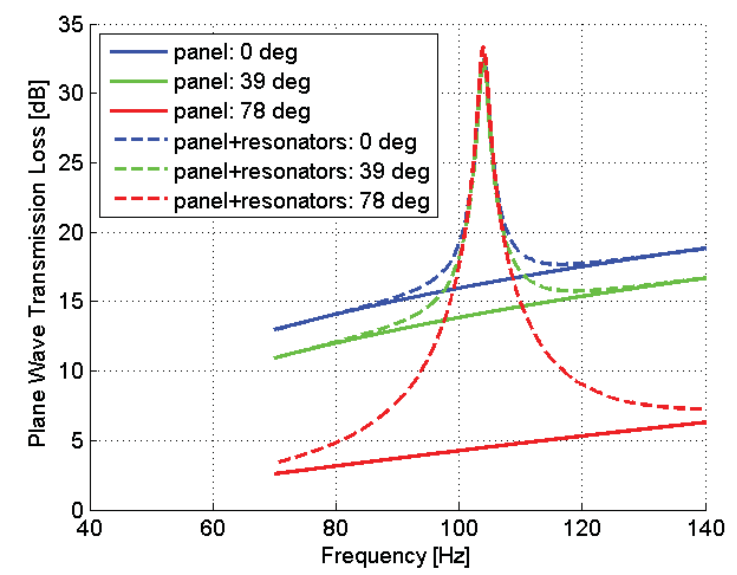

Figure 6: Plane wave transmission loss versus frequency. The solid lines are for the baseline panel without inlets, and the dashed curves are for the panel with inlets.

The dashed lines in Figure 6 show the transmission loss through the same panel with open inlets to the acoustic resonators. In this case, the acoustic cavities in the core become resonators with a natural frequency of $104 \mathrm{~Hz}$. Near resonance, the transmission loss is increased by $17 \mathrm{~dB}$ at normal incidence and by more than $28 \mathrm{~dB}$ at an incidence angle of 78 degrees.

It is interesting to note that at resonance, the transmission loss of the panel with open inlets is independent of incidence angle. This is true despite the fact that the transmission loss through the baseline panel (without inlets) does vary with incidence angle. To understand why, consider the fact that at normal incidence, the specific impedance of the panel is an order of magnitude larger than the specific acoustic impedance of air. Because of the large impedance mismatch, most of the incident energy is reflected and therefore the transmission loss is relatively high. However, as the angle of incidence increases, the specific acoustic impedance of the gas at the surface of the panel increases significantly, while the impedance of the panel

\section{5}

This material is declared a work of the U.S. Government and is not subject to copyright protection in the United States. Approved for public release; distribution is unlimited. 
remains fixed. Therefore the impedance mismatch between the fluid and structure is reduced at high angles of incidence. This leads to better sound transmission and therefore lower transmission loss through the panel. However, near the acoustic resonance the specific impedance on the transmitted side of the panel is dominated by the resonator. Since the resonator is locally reacting, which means that its impedance is independent of angle of incidence, the combined transmission loss of the panel with embedded resonators is largely independent of incidence angle near resonance.

It is also instructive to consider the behavior from an energy perspective. Table 3 compares the incident, reflected, transmitted, and dissipated sound power at resonance for the panel with and without inlets. The values are calculated assuming a plane wave excitation at normal incidence. While the incident sound power is identical for both cases, the power transmitted through the panel with inlets is approximately 40 times (or $16 \mathrm{~dB}$ ) smaller than the power transmitted through the panel without inlets. This benefit can be attributed to an increase in both the power reflected from the panel and the power dissipated in the resonators. However, it should be noted that the power dissipated in the resonators is two-orders of magnitude less than the reflected power. Therefore the change in the reflected power has a much larger impact on transmission than the change in dissipated power.

Table 3: Normalized sound power at resonance (104 Hz).

\begin{tabular}{|c|c|c|}
\hline Sound Power & panel & panel + resonators \\
\hline Incident & 1 & 1 \\
\hline Reflected & $9.77 \times 10^{-1}$ & $9.97 \times 10^{-1}$ \\
\hline Transmitted & $2.24 \times 10^{-2}$ & $5.05 \times 10^{-4}$ \\
\hline Dissipated & $8.55 \times 10^{-4}$ & $2.52 \times 10^{-3}$ \\
\hline
\end{tabular}

Figure 7 shows the diffuse field transmission loss, which is essentially a weighted average over all angles of incidence. Near resonance, the resonators improve the diffuse field transmission loss by more than $22 \mathrm{~dB}$. While it may not be intuitive, adding holes (or ports) through the facesheet significantly increase the transmission loss of the system around the natural frequency of the embedded resonators. Before studying the parameters that affect performance, it is first helpful to consider a physical explanation for this behavior.

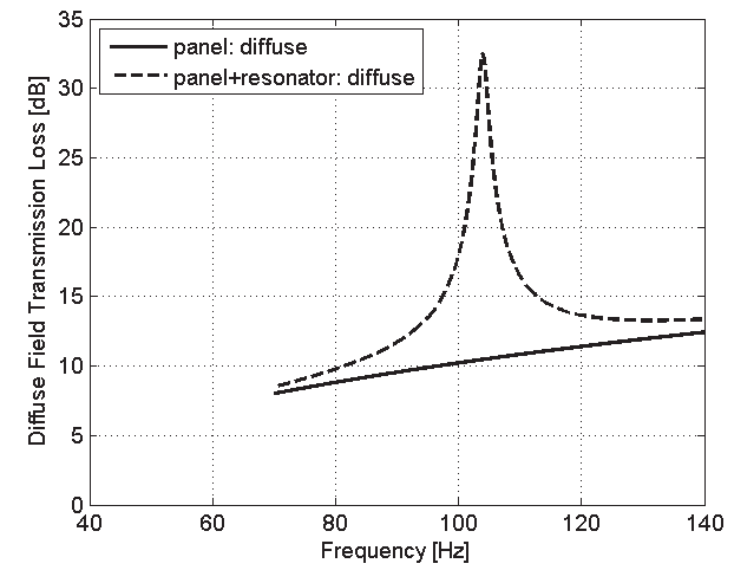

Figure 7: Diffuse field transmission loss for a panel with (dashed line) and without (solid line) inlets.

\section{Physical explanation}

An increase in the transmission loss implies that less sound is radiated from the panel when the inlets are open. To understand why this occurs consider Figure 8, which shows the normal component of the acoustic intensity on a cut plane $10 \mathrm{~cm}$ below the panel. In this case, positive values indicate that energy is flowing towards the panel, while negative values indicate that energy is flowing away from the panel. Notice that most of the panel is blue, which indicates that the energy is flowing away from the panel. However near the inlets, the net intensity is positive. Since the total sound power radiated by the structure can be found by integrating the intensity over the surface of the cut plane, the positive regions around the inlets reduce the overall sound power radiated from the panel. In other words, at the resonance, the ports radiate out-of-phase with the structure resulting in less total radiation due to the destructive interference.

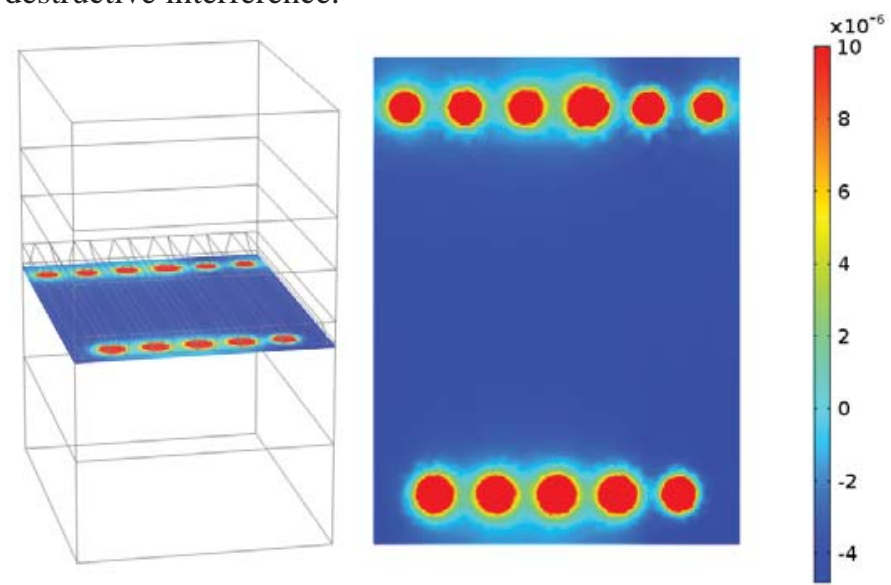

Figure 8: Acoustic intensity $\left(\mathrm{W} / \mathrm{m}^{2}\right)$ on a cut plane $10 \mathrm{~cm}$ below the panel.

\section{Parameter study}

The impacts of chamber length, cavity area, and inlet area on transmission loss are considered next. Since the

\section{6}

This material is declared a work of the U.S. Government and is not subject to copyright protection in the United States. Approved for public release; distribution is unlimited. 
transmission loss through the panel with open inlets is largely insensitive to the angle of incidence, the parameter study is performed at 0 degrees. The following plots show the baseline transmission loss without inlets, along with the nominal response with inlets. The nominal response is found using the parameter values given in Table 2. Individual parameters are then varied independently by some percentage of the nominal value. In all cases, the overall size and mass of the panel are held fixed. The only changes are associated with the resonators embedded in the panel.

Figure 9 shows the effect of resonator length on transmission loss. The length of the resonators clearly affects the natural frequency of the device. Specifically, decreasing the length increases the natural frequency. This trend is predicted by both Eqs. (6) and (9) for quarter wavelength and Helmholtz resonators, respectively. Notice that while the peak response increases with frequency, this is due to the fact that the transmission loss of the bare panel increases with frequency. The relative transmission loss, or difference between the dashed line and solid line, is very similar for the three lengths considered.

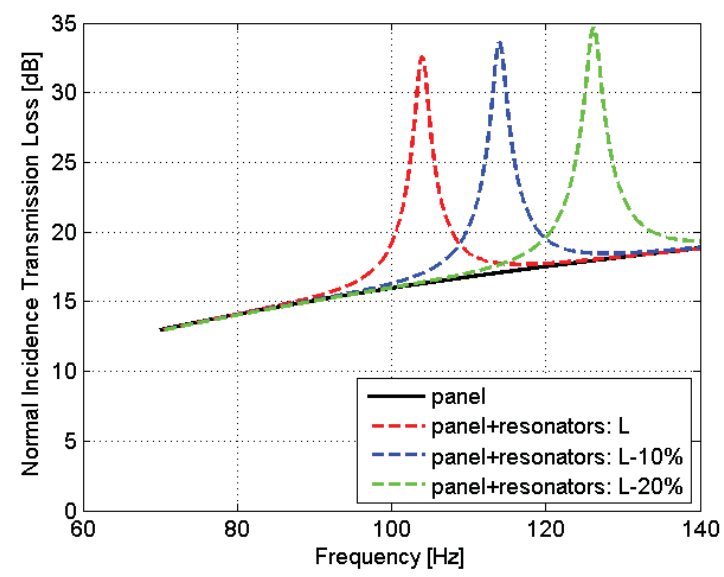

Figure 9: Effect of resonator length on normal incidence transmission loss.

Figure 10 shows the effect of the cavity cross-sectional area on transmission loss. While there is a noticeable shift in the natural frequency, the effect is less pronounced than when the resonator length was varied. Specifically, the natural frequency of the resonators increases as the area is reduced. This trend is not predicted by Eq. (6) since the natural frequency of a quarter wavelength resonator is independent of cavity area; however it is predicted by the Helmholtz resonator equation, Eq. (9). In addition to shifting the natural frequency, changing the cavity area also affects the peak response. This is because the cavity losses depend on the radius of the duct. Specifically the losses increase as the cross-sectional area is reduced. Based on Figure 10, it appears that increasing the cavity area could be used to simultaneously increase performance and reduce the natural frequency of the resonators. One way to increase the cavity area without affecting other design parameters is to increase the overall panel thickness.

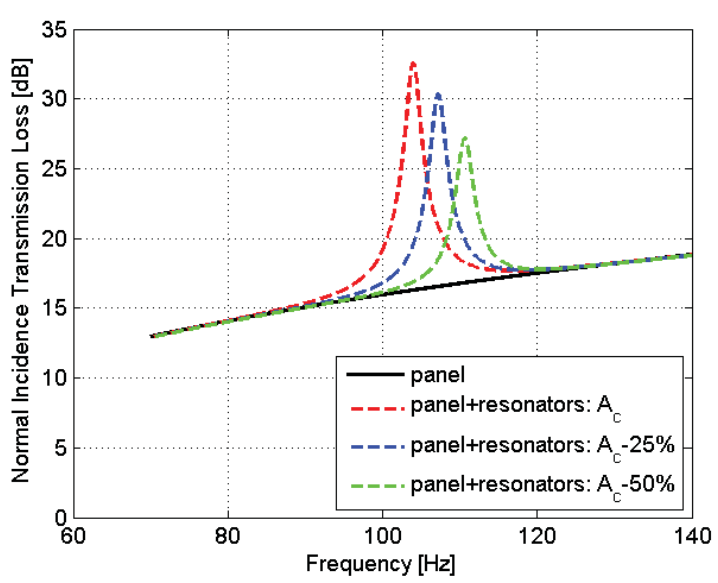

Figure 10: Effect of resonator cross-sectional area on transmission loss.

Figure 11 shows the effect of inlet area variations on transmission loss. Unlike the previous two parameters, reducing the inlet area decreases the natural frequency of the resonator. Once again, this trend is accurately predicted by the Helmholtz resonator equation. In addition to shifting the natural frequency, reducing the inlet size also increases the losses in the inlet. This is due to the fact that the inlet attenuation coefficient is inversely proportional to the effective radius of the inlet. Therefore the relative transmission loss is reduced as the inlet area gets smaller.

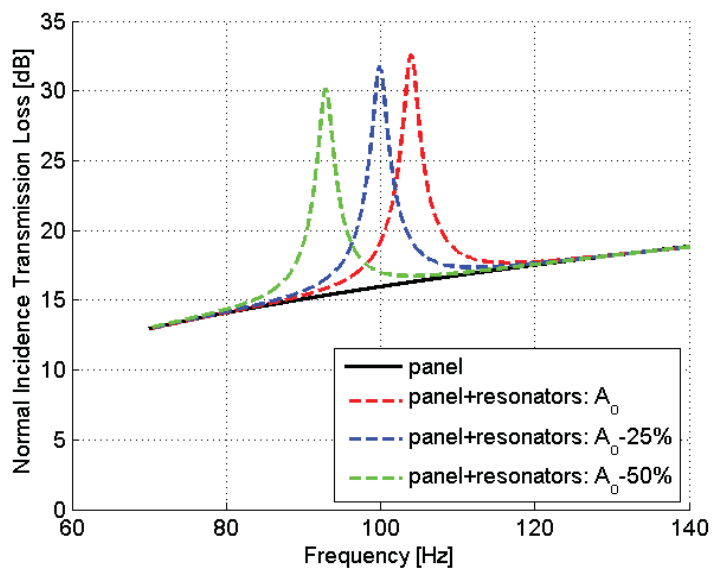

Figure 11: Effect of inlet area on transmission loss.

While it is useful to consider the effect of individual parameter variations, it is also valuable to compare combinations of parameters that are constrained to have the same natural frequency, $f_{n}$, and the same resonator volume per unit area, $V_{s}=A_{c} L N_{r} / S$. As the equation implies, $V_{s}$ is the total volume of the resonators divided by the surface area of the panel. The results are shown in Table 4. Notice that the peak transmission loss at resonance is nearly identical for all designs. This suggests that for a given natural frequency, the overall

7

This material is declared a work of the U.S. Government and is not subject to copyright protection in the United States. Approved for public release; distribution is unlimited. 
performance is largely determined by the resonator volume per unit area.

Table 4: Transmission loss at resonance for several parameter combinations.

\begin{tabular}{|c|c|c|c|c|c|}
\hline Parameter & Nominal & Case 1 & Case 2 & Case 3 & Case 4 \\
\hline$m_{S}\left(^{*}\right)$ & 1 & 1 & 1 & 1 & 1 \\
\hline$S\left(^{*}\right)$ & 1 & 1 & 1 & 1 & .75 \\
\hline$N_{r}\left({ }^{*}\right)$ & 1 & 1 & 0.333 & 2 & 1.11 \\
\hline$L\left(^{*}\right)$ & 1 & 0.9 & 1 & 1 & 0.9 \\
\hline$A_{c}\left(^{*}\right)$ & 1 & 1.11 & 3 & 0.5 & 0.75 \\
\hline$A_{o}\left({ }^{*}\right)$ & 1 & 0.632 & 3 & 0.5 & 0.431 \\
\hline$f_{n}(\mathrm{~Hz})$ & 104 & 104 & 104 & 104 & 104 \\
\hline$V_{S}(\mathrm{~m})$ & 0.041 & 0.041 & 0.041 & 0.041 & 0.041 \\
\hline $\begin{array}{c}\text { Peak TL } \\
(\mathrm{dB})\end{array}$ & 32.6 & 32.3 & 32.6 & 32.6 & 32.3 \\
\hline * relative value with respect to Table 2 & & \\
\hline
\end{tabular}

Finally, Figure 12 shows the impact of changing the resonator volume per unit area on transmission loss. While there is no effect on the natural frequency of the resonators, the amplitude and width of the peak are reduced as $V_{S}$ is reduced. To clarify this relationship, Figure 13 shows the relative transmission loss (i.e. the difference in transmission loss between the baseline panel and the panel with inlets) as a function of resonator volume per unit area. The red marker indicates the nominal design point. For this configuration, a logarithmic function can be used to describe the relationship between relative transmission loss and resonator volume per unit area as long as $V_{s} \geq 0.03$,

$$
\Delta T L=17.6 \log _{10}\left(V_{S}\right)+40.7
$$

Using this expression, it can be shown that varying the resonator volume per unit area by a factor of 2 will change the transmission loss by approximately $5 \mathrm{~dB}$. In summary, when the natural frequency of the resonators is fixed, performance is determined primarily by the resonator volume per unit area. Increasing the volume improves performance. Therefore, sandwich panel designs with embedded resonators should attempt to maximize the use of the interior core volume.

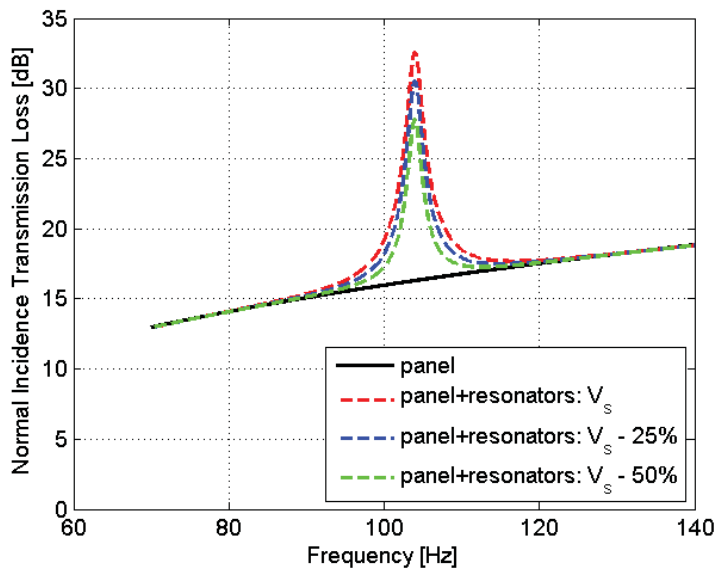

Figure 12: Effect of resonator volume per unit area on transmission loss.

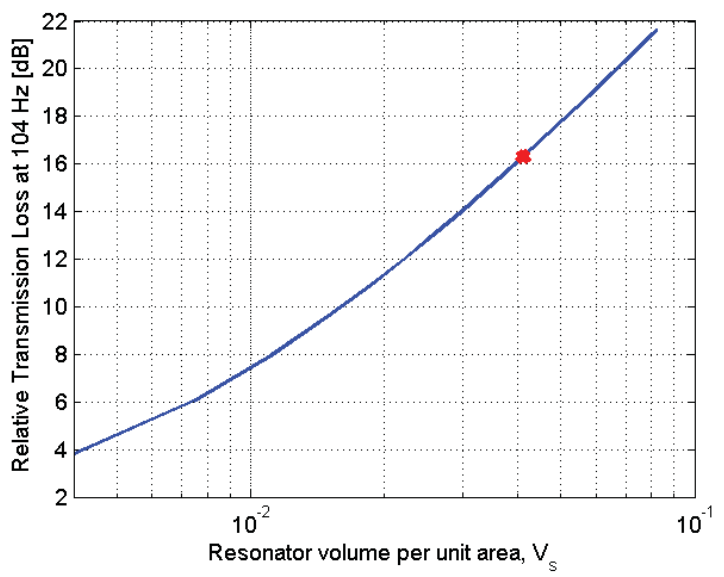

Figure 13: Relative transmission loss as a function of resonator volume per unit area. The red marker denotes the nominal design.

\section{CONCLUDING REMARKS}

While resonators are commonly used to augment absorption, this paper shows that when integrated within a panel, resonators can also be used to improve the sound transmission loss of the structure. Specifically, this paper describes analytical and numerical models of the sound transmission loss through a sandwich structure with acoustic resonators integrated in the core. Predictions generated using both types of models compare very well and show that resonators can significantly increase the transmission loss of the panel around their natural frequency. In one configuration, the diffuse field transmission loss is improved by more than $22 \mathrm{~dB}$ at $104 \mathrm{~Hz}$. This benefit is achieved with no added mass or volume. Simulations demonstrate that at resonance, the sound radiation from the resonators is out-of-phase with the structure. This results in destructive interference and less overall sound radiation from the panel. The impact of several design parameters is also considered, including cavity length, cavity area, and inlet area. Based on the parameter study, it appears that the performance of the system is ultimately

8

This material is declared a work of the U.S. Government and is not subject to copyright protection in the United States. Approved for public release; distribution is unlimited. 
dependent on the total volume of the resonators. Therefore, it is desirable to maximize the volume of the resonators within a given structure.

Future work will focus on experimentally validating the models. There are also plans to generate larger vehicle level models and capture the effect due to absorption. Optimizations could then be performed to maximize the vibroacoustic performance of the structure for a given application.

\section{REFERENCES}

1. Bies, D. A, and Hansen, C. H., 1996, Engineering Noise Control, Spon, London.

2. Parrott, T. L., and Jones, M. G., 2012, "Uncertainty in Acoustic Liner Impedance Measurement and Prediction," NASA/TP-2012-215653.

3. Estéve, S. J., and Johnson, M. E., 2002, "Reduction of sound transmission into a circular cylindrical shell using distributed vibration absorbers and Helmholtz resonators," Journal of the Acoustical Society of America, 112(6), 2840-2848.

4. Kuntz, H. L., Gatineau, R. J., Prydz, R. A., \& Balena, F. J., 1991, Development and testing of cabin sidewall acoustic resonators for the reduction of cabin tone levels in propfanpowered aircraft, NASA CR 4388.

5. Lane, S. A., Richard, R. E., \& Kennedy, S. J., 2005, "Fairing noise control using tube-shaped resonators," Journal of Spacecraft and Rockets, 42(4), 640-646.

6. Lane, S. A., Henderson, K., Williams, A., and Ardelean, E., 2007, "Chamber core structures for fairing acoustic mitigation,” Journal of Spacecraft and Rockets, 44(1), 156163.

7. Li, D., and Vipperman, J. S., 2006, "Noise control of mock-scale chambercore payload fairing using integrated acoustic resonators," Journal of Spacecraft and Rockets, 43(4), 877-882.

8. Chang, W. S., Ventsel, E., Krauthammer, T., and John, J., 2005, "Bending behavior of corrugated-core sandwich plates,” Composite Structures, 70(1), 81-89.

9. Cummings, A., 1975, "Sound transmission in 180 degree duct bends of rectangular section,” Journal of Sound and Vibration, 41(3), 321-334.

10. Panton, R. L., and Miller, J. M., 1975, "Resonant frequencies of cylindrical Helmholtz resonators,” Journal of the Acoustical Society of America, 57(6), 1533-1535.

11. Blackstock, D. T., 2000, Fundamentals of physical acoustics, John Wiley \& Sons.

12. Pierce, A. D., 1989, Acoustics: An introduction to its physical principles and applications, Acoustical Society of America.

13. Kinsler, L. E., Frey, A. R., Coppens, A. B., \& Sanders, J. V., 2000, Fundamentals of acoustics, $4^{\text {th }}$ edition, John Wiley \& Sons.

14. Fahy, F., and Gardonio, P., 2007, Sound and structural vibration: radiation, transmission, and response $2^{\text {nd }}$ Edition, Academic Press, Oxford.
15. COMSOL tutorial, 2013, "Acoustic transmission loss through periodic elastic structures,” Model Database 1974.

This material is declared a work of the U.S. Government and is not subject to copyright protection in the United States. Approved for public release; distribution is unlimited. 